\title{
Effect of Low Frequency Electrical Current on the Biophysical and Molecular Properties of Cancer Cells
}

\author{
Amit Sengupta ${ }^{1 *}$, Sanjay Gupta ${ }^{2,3 \#}$, Asmita Sharda ${ }^{2,3}$, Hemant $S^{1}$ and Arvind Ingle \\ ${ }^{1}$ Bioengineering and Gynae-Colposcopy Lab, Advanced Centre for Treatment, Research and Education in \\ Cancer, Tata Memorial Centre, Kharghar, Navi Mumbai, India \\ ${ }^{2}$ Epigenetics and Chromatin Biology Group, Gupta Lab, Cancer Research Institute, Advanced Centre for \\ Treatment Research and Education in Cancer, Tata Memorial Centre, Kharghar, Navi Mumbai, India \\ ${ }^{3}$ Homi Bhabha National Institute, Training School Complex, Anushakti Nagar, Mumbai, India \\ ${ }^{4}$ Laboratory Animal facility, Advanced Centre for Treatment, Research and Education in Cancer, Tata \\ Memorial Centre, Kharghar, Navi Mumbai India \\ ${ }^{\#}$ Co-corresponing author
}

*Corresponding author: Dr. Prof Amit Sengupta, Independent Consultant in Obstetrics and Gynecology-Oncology and Professor Bioengineering, 301-52, Seawoods Estates, Nerul, Navi Mumbai, Maharashtra, India-400706, Tel: +91-996-9011800; +91-982-032-9861

\begin{abstract}
Background: Different cells and tissues are known to exhibit varied electromagnetic, electrical and molecular properties. During the repair process, cancer cells as well as normal proliferating cells have higher transmembrane potential than healthy cells. Since the dielectric properties are frequency dependent, applying varying frequencies of current can alter the transmembrane ionic flux of any conductive cell. This, in turn, can generate heat via the joule effect. Thus, it might be possible to alter the tumor microenvironment using low frequency electric current.

Methods: The present study was designed to analyze the effect of low frequency AC (Alternating Current) on molecular properties of cells and understand its effect on tissue bioimpedance. An in vitro and ex-vivo study was conducted in mouse model of mammary tumor and compared with a phantom model. Low frequency AC sinusoidal current of constant amplitude was generated by the sine wave oscillator (1-5 mA and frequency of $100 \mathrm{~Hz}$ ) and the bioimpedance values were recorded with the help of two needle or sensing electrodes. Molecular changes were also documented from the samples subjected to low frequency current. Protein levels of Heat Shock Protein 90 (HSP90) and beta-actin were also analyzed to evaluate any thermal effects. Additional information about genome stability and chromatin de-condensation was assessed by levels of
\end{abstract}

histone Post Translational Modifications (PTMs) $\mathrm{yH} 2 \mathrm{AX}$ and $\mathrm{H} 3 \mathrm{~K} 9 \mathrm{Ac}$, respectively.

Results: An increase in tissue bio-impedance (decrease in the capacitance or conductance) value was observed with an increase in the frequency of the applied current. The bio-impedance value for normal tissue was found to be in the range $17-27 \Omega \mathrm{k}$ at $100 \mathrm{~Hz}$ (applied for a period of 1 minute) and it increased to the range of $27-37 \Omega \mathrm{k}$ at $1 \mathrm{kHz}$. Additionally, lower impedance value of (range 16-22 $\Omega \mathrm{k}$ ) at $100 \mathrm{~Hz}$ and (range 24-33 $\Omega \mathrm{k}$ ) at $1 \mathrm{kHz}$ was observed for cancer tissue. At the morphological level, some cell swelling in tissue samples and cell isolates was observed at low frequency current of $100 \mathrm{~Hz}$, possibly contributed by heating. An increased cell swelling, shrinkage or rupture and disintegration were noted upon treatment with a slightly higher frequency $(1 \mathrm{kHz})$ when applied for more than 1 minute. Low levels of beta-actin and HSP90 proteins suggested cell surface derangement and/or apoptosis in the in-vitro model whereas their high levels were observed in the ex-vivo model. Interestingly, no epigenetic alterations suggestive of genotoxicity were observed.

Conclusion: The present study generated better understanding of the interactions between tissue/cell \& electrical field and responses. These responses were in the form of bio-impedance and morphological change, heat production, changes in DNA and proteins etc. There is a need to generate extensive tissue and cell specific data

\footnotetext{
Citation: Sengupta A, Gupta S, Sharda A, Hemant S, Ingle A (2021) Effect of Low Frequency Electrical Current on the Biophysical and Molecular Properties of Cancer Cells. Int J Cancer Clin Res 8:145. doi. org/10.23937/2378-3419/1410145

Accepted: February 23, 2021: Published: February 25, 2021

Copyright: (C) 2020 Sengupta A, et al. This is an open-access article distributed under the terms of the Creative Commons Attribution License, which permits unrestricted use, distribution, and reproduction in any medium, provided the original author and source are credited.
} 
on the impedance value, thermal effects and cell specific aneuploidy and epigenetic modifications. The results obtained from such large well controlled in-vitro and in-vivo experiments under normal physiological conditions can then be exploited to develop label free cancer diagnostics \& therapeutics.

\section{Keywords}

Low frequency current, Thermodynamics, Label free biomarker, Theronostics, Tumor, The tumor microenvironment

\section{Introduction}

Cancer is a disease characterized by an abnormal and uncontrolled division of cells that could also have invasive properties. It involves undiminished cellular proliferative potential, loss of differentiation, angiogenesis, metastasis and invasion. The proliferating cells interact constantly with their surrounding stromal microenvironment [1]. Mathematically, a continuous time deterministic multi-event theoretical model can explain such a phenomenon of birth, death and mutation over a period of time. It basically describes the mean behavior of the evolutionary process where all the three events take place at every point in time with a predictable outcome [2]. In this context, Rashevsky-Turing theory is also important, which puts forth a basic question of morphogenesis. The theory questions about movement of cell aggregates from an unstable state to a well defined asymptotically stable state and vice-versa. However, entropy relates to the independence of the tumor cell and the switch in its physiology, i.e., from subservience to the organism to its own independent Darwinian evolution [3,4]. Any perturbatión in demand and supply balance of substrates and energy (oxygen, nutrient, co-factors, electro-magnetic field) leads to an unsteady state. This scenario is time-dependent which may either revert back to a normal steady state (reversible) or grow exponentially to initiate the process of cancer or undifferentiated uncontrolled growth (irreversible) upto a limit of asymptotic state. The process acts through altered pathways of DNA synthesis, DNA damage and repair mechanism and cell death that are more evident in dividing cells [3-15]. A recent animal model-based study by our group highlighted tumor growth and progression to be significantly affected by the altered physical (Hyper-Baric Oxygenation (HBO)) stress, thereby causing perturbation in the tumor microenvironment $[16,17]$. Similar findings were also observed in one of the clinical study [6]. In biophysical or bioelectrical terms, normal proliferating cells and cancer cells behave quite differently with respect to depolarization, suggesting that it is more positive inside the cytoplasm of a cancer cell compared to a normal cell. The dividing cancer cells have higher resting potential across cell membrane $(-15 \mathrm{mV})$ compared to trans-membrane resting potential (-70 to-100 $\mathrm{mV}$ ) of a normal cell [18]. Interestingly, the transmembrane potential of a normal repairing cell returns to normal resting polarized state following repair process, but a cancer cell continues to maintain a higher transmembrane potential. Different cells have different ions and biomolecules; even the environment within the cytoplasm is jelly-like, with water acting as a sluggish fluid medium [19] whereas in cancer cells, this water becomes more mobile [20].

The dielectric properties of cells are also frequency dependent [21] e.g., $\alpha$-dispersion occurred at a few $\mathrm{kHz}$ and with increasing frequency, extra dispersions occur at a frequency ranging from tens of $\mathrm{kHz}$ to tens of $\mathrm{mHz}$. This is referred to as the $\beta$-dispersion [22]. These dielectric dispersions indicate that the polarization process in the cells is mediated by different mechanisms and that one mechanism is lost at a specific range of frequencies. Both the dispersions at low frequencies are mainly caused by the structure of the cell, more specifically the cell membranes and the free ions within and outside the cell [23]. Cell capacitance also showed an exponential decaying behavior versus frequency, that is as the frequency is increased, the capacitance gets decreased rapidly or impedance increased and vice-versa. The cancer cell is known to exhibit lower capacitance value compared to normal cells of different tissue origin [23]. An important implication of this finding is that even at Direct Current (DC) voltages (as well as frequencies up to $1 \mathrm{mHz}$ ) manipulation of the field strength in the cell membrane can be achieved [24]. When an electromagnetic field (DC/low frequency AC current) is applied to the cell (circuit) or cellular resistive components, lattice heat will develop due to the loss of kinetic energy by the accelerated electrons due to collision with the atoms. This is nothing but the resistance (impedance) that corresponds to an increase in lattice heat, which is irretrievable. By Joule's law, we can see that the heat energy dissipated by current (I) flowing through a resistor of resistance $\mathrm{R}$ is $\mathrm{H}=I^{\wedge} 2^{*} \mathrm{R}$. This energy in the crystal lattice (tissue) induces thermal movement of electrons that will be in a random direction. This randomness can be defined as entropy. The change in entropy is denoted by $d S=d Q / T$, where $d Q$ is the change in heat energy between two time intervals and $T$ is the final temperature. Thus, by applying an external electromagnetic field (DC/Low frequency), we can further increase in the entropy of the system. Laws governing thermodynamics state that there exist two energy states and entropy between two closely inter-dependent systems. Entropy is a measure of randomness resulting from the increase in heat energy supplied to a system. Cancer initiating processes follows the second law of thermodynamics where energy/heat flows towards the disturbed area (cancer entropy) to supply much needed energy. This continues until the required demand is met through aerobic pyruvic acid metabolic pathway and subsequently by aerobic glycolysis (Warburg hypothesis), whereby a new threshold of steady state or a higher state of entropy is reached.

Consequently, when the normal energy equilibrium 
(cell charge/dielectric property) is disturbed, many exogenous (e.g., electromagnetic field, oxygen, nutrients) and endogenous factors may potentiate mutagenic effect on cells during cell division phase. Hyperthermia Therapy (HT) for longer duration may lead to Vascular Endothelial Growth Factor (VEGF) and Hypoxia Inducible Factor (HIF-1) up-regulation, thereby creating onco-thermia (reversible or irreversible energy state transformation) with low voltage low dose electrical current, via joule effect can be a better and safer modality $[16,17]$. DC electric field is also known to cause tissue repair (electric field polarizes charged cell surface molecules). At extremely low frequencies, electric field stimulates excitable tissues through membrane depolarization, stimulates bone growth and accelerates fracture healing [25]. As the electric field frequency increases, the stimulatory effect disappears, ending in the so-called thermal effect, when tissue heating becomes a dominant event [26]. Tumor Treating fields (TT Fields) is a noninvasive, regional antimitotic treatment modality that has been approved for the treatment of recurrent glioblastoma by the U.S. FDA and has a CE mark in Europe. TT Fields therapy delivers low-intensity (1-3 V/cm), intermediate-frequency $(100-300 \mathrm{kHz})$, alternating electric fields to the tumor using transducer arrays placed on the skin around the region of the body containing the tumor [27]. Low Dose Alternating Electric Current (LDAEC) demonstrates efficacy against C4-2B and LuCaP35 xenograft while causing no harm to nerves and blood vessels. These results warrant further investigations into the use of LDAEC as a treatment for CaP [28]. This study hypothesizes that the successes of studies of Direct Current (DC) against cancer tumors is due to the fact that $D C$ inhibits the ribonucleotide reductase enzyme, which is necessary for cancer cell growth [29].

In this context, it is important to discuss the recent in vivo studies on the effects of non-thermal irreversible electrolytic electroporation (E2) in cancer therapeutics under different experimental conditions. It involves giving short and high voltage direct current pulses to the tumor, unlike in our study where we have utilized low frequency AC current. Klien, et al. showed that cell killing efficiency actually depended upon a combination of factors such as electrolysis, reversible electroporation and additional thermal effects [30,31]. The process of electroporation has been shown to induce cell death and cell regeneration through inflammatory immuno-modulatory response, pyroptosis, necroptosis and programed necrosis [32,33]. Irreversible electroporation has also been utilized in advanced cancers as an ablation therapy tool with some survival benefits but tissue heterogeneity, persistence of viable malignancy and epigenetic modifications/genotoxicity continue to challenge the scientific community. The recurrence rate will remain high unless cell-specific safe and optimal doses are titrated and applied to specific cells/tissue types according to the size of the tumor under electropora- tion. Concurrently, one needs to examine its thermal effects on the tissue, which is a subject of controversy, also patient selection and size, type and grade of the tumor $[34,35]$. Therefore, on the basis of all the existing knowledge, it can safely be assumed that different cells and tissues exhibit different thermodynamic, electromagnetic and electrical properties. The electromagnetic forces and heat generated by the living cells and tissues also plays a significant role in cell proliferation and function $[5,18,36]$. Studies on the biophysical and molecular behavior in response to low frequency electrical current may open up the possibility to develop a label free diagnostic biosensor for cancer screening as well as an onco-thermia based treatment modality.

In this study, the experiments designed were based upon the hypothesis that there exist two energy states and an ionic balance between two closely inter-dependent systems, acting in a synchronous manner. Any disturbance or perturbation might trigger the process of an abnormal proliferation of endothelial cells and adjoining mutant cells by gaining or losing resting potential and heat energy (entropy). The reversal of the process of entropy flow by low frequency electrical current may either enhance the process of apoptosis leading to tumor regression or it may also induce a state of dormancy. A characterization of the electrical response of a cell cluster and tissues i.e., the effect may be performed of electric stress on normal and cancer cells and tissues by measuring their impedance (resistance) values using simple four needle electrode system. For therapeutic purpose, we estimated and determined the value/ range of low frequency current (external electromagnetic field) that can induce ionic disruption in the cell membrane and cause entropy rise capable of causing permanent cellular injury/cell deaths specifically in cancer cells without having any genotoxicity or epigenetic effects. The DNA damage, cell lysis or deaths and other epigenetic and surface protein changes triggered by the electrical energy were also studied.

\section{Materials and Methods}

\section{In vitro/ex vivo mammary tumor model}

Experiments and model generation were carried out in Laboratory Animal Facility (LAF) at ACTREC in strict accordance with the recommendations of the Committee for the Purpose of Control and Supervision of Experiments on Animals (CPCSEA), Ministry of Environment and Forests, Govt. of India. The protocol was approved by the Institutional Animal Ethics Committee (IAEC) of Tata Memorial Centre-Advanced Center for Treatment Research and Education in Cancer (Proposal No. 10/2012 dated, 12-08-2012). All efforts were made to minimize suffering during the study. An inbred $\mathrm{C} 3 \mathrm{H}$ strain with agouti coat color was used for this study. Genetically identical six to eight-week-old inbred $\mathrm{C} 3 \mathrm{H}$ (Agouti) female mice were housed in cages with access 
to food and water ad libitum and exposed to 12-hour light dark cycle. The breeding system adopted in our animal facility was $1: 1$ brother sister mating till 20 generation to achieve isogenicity. The isogenicity has been further verified by the animal house facility with biochemical markers (Hbb, Car-2, Idh, Mod), skin grafting and molecular markers (microsatellite) apart from regular health monitoring. Mammary tumors were induced using $0.1 \mathrm{ml}$ subcutaneous injection of cell suspension ( 20 million cells $/ \mathrm{ml}$ ) in mouse mammary fat pad. A tumor cell suspension was prepared from aseptically excised spontaneous mammary tumor. Daily inspection of the induced mice was done for food, water, bedding, comfort and appearance of the tumor nodule under all aseptic precaution by the same research fellow to minimize the error and reduce inter personal variation. The tumor measurements were noted from the day the nodule was palpated. The mice were subjected to experimental intervention from the time. The nodule size measured between 5-10 mm in diameter. Macroscopic tumors were evident by day 60 (median value/mean value) following tumor induction-latent period. Mammary tumor from small mice was excised (one group immediately after sacrifice to simulate ex-vivo condition and the second group within one hour of natural death (in vitro condition). The tumor was cut into four parts - first small part was put under experimentation immediately and then freeze dried for molecular study; the second part was immediately freeze dried, one part of which was subsequently made into sections of equal size using cryostat-microtome for further experimentation with low frequency currents (thawed at room temperature) and then again freeze dried and kept for bio-molecular studies. The third freshly excised part was kept as control for morphological and bio-molecular studies, the fourth part was freeze dried immediate but later thawed later at room temperature was kept as control for molecular study (re-freeze dried).

\section{In vitro Phantom models}

\section{i. Cell suspension \\ ii. Microsphere}

\section{Cell suspension preparation:}

(a) Freshly prepared tumor and normal tissue samples from freshly sacrificed animals were snap frozen and put in PBS in order to prepare cell suspensions. $50-70 \mathrm{mg}$ piece of the tissue was aseptically collected in a sterile micro-centrifuge tube. Digestion of the tissue piece was performed in $300 \mu \mathrm{l}$ digestion buffer containing $18 \mu \mathrm{l}$ of proteinase $\mathrm{K}\left(20 \mathrm{mg} / \mathrm{ml}\right.$ in $1 \mathrm{X} \mathrm{TE}$ buffer) at $50{ }^{\circ} \mathrm{C}$ in a roller mixer incubator for 5-6 hours. This was followed by centrifugation at $200 \times \mathrm{g}$ for 10 minutes at room temperature. Pellet was collected and re-suspended in Phosphate Buffered Saline (PBS).
(b)Cell suspension from fixed tissue or tissue ribbon - Paraffin embedded tissues collected from the mice was made in to ribbon slices of $5 \mu \mathrm{m}$ size using microtome. The tissue ribbon was taken in a micro-centrifuge tube, $1 \mathrm{ml}$ of xylene was added and incubated for 10-15 minutes. It was then centrifuged at $300 \times \mathrm{g}$ at room temperature for 10 minutes. $1-2 \mathrm{ml}$ of absolute alcohol was added to the pellet to remove the xylene and incubated for 10-15 minutes followed by centrifugation at $300 \times \mathrm{g}$ at room temperature for 10 minutes. Another wash of $70 \%$ alcohol was given to the pellet to remove the xylene content completely, followed by washing with distilled water to remove the alcohol content completely. $2 \mathrm{ml}$ of $0.1 \%$ trypsin was added to the pellet and kept it on the sand bath overnight. Next day, the sample was centrifuged at $300 \times \mathrm{g}$ at room temperature for 10 minutes. The pellet was collected and re-suspended in pellet in TBS.

Microsphere phantom model: This model was developed to reproduce biological cells in morphology and function as closely as possible. Small spherical particles with diameter in the micron range are here used as simulating phantom for the cell. $50 \mathrm{ml}$ of $70 \%$ ethanol was mixed well with equal volume of Dichloromethane (1:1 ratio). This was added dropwise to $100 \mathrm{ml}$ Sodium Dodecyl Sulphate (SDS) and centrifuged at $200 \times \mathrm{g}$ at room temperature. After the preparation, a drop was put on a slide and mounted for the digital imaging using confocal microscope. Size measurement was done using LSM image software.

\section{Bio-impedance measurement}

The experimental model developed for this study was based upon our previous studies on electrical impedance tomography and tumour microenvironment modulation [37-39]. The entire experiment was divided into 2 sets- a) Experimental group-whereby microsphere, cell suspensions, mammary tumor and normal tissue samples were exposed to current of different frequencies and b) Control group- where tissues were not exposed to current. Tissues were taken i) Post-natural death ( $<1$ hours - in vitro; advanced stage of cancer) and ii) Post sacrifice for the mice (fresh samples - ex vivo-advanced stage but not terminal).

\section{Heat effects for a reversible reaction (Q)}

These values were calculated by the formula $=T \Delta S$ where, $\Delta S$ is the entropy change of this reaction and $T$ temperature in Kelvin. It is difficult to measure the entropy if the steady state reached by the cancer cell (tissue-microenvironment) is disturbed. We expected to raise the surrounding tissue temp to 40 degree by low frequency current assuming that the transformation is reversible for surrounding normal cells at a lower frequency. 


\section{Directly Applied Low Frequency AC (LFAC) current}

Low frequency AC sinusoidal current of constant amplitude generated by the sine wave oscillator (1-5 mA and frequency of $100 \mathrm{~Hz}$ ) was passed through the tissue soaked in buffer (post death $(<1$ hour - in vitro) and sacrificed-ex vivo (fresh) with the help of two needle current electrodes placed directly on the tissue. Voltage signal developed along the current path was recorded with the help of another two needle or sensing electrodes.

\section{Indirect Applied Low frequency AC (LFAC) Current (at $100 \mathrm{~Hz}, 1 \mathrm{kHz}$ )}

Electrodes were placed on the electrolyte solution (electrode-electrolyte layer) in a chamber where a tissue was placed. It raised the temperature of the solution by creating heating effect. The following steps were followed for this protocol-

(a) Needle electrode was placed directly on the tissue (direct low frequency AC current) or placed in the electrolyte solution (saline/ $\mathrm{KCl}$ buffer holding the tissue) creating tissue electrical field. Frequency dampening was seen due to $\mathrm{R}$ of the electrolyte medium that is saline/Brine solution.

(b) Impedance meter consisting of the chamber (where the samples were submerged) comprises of four electrodes being used to measure the impedance. Out of four electrodes, a pair of electrode was connected to signal generator of the $\mathrm{Dr}$. DAQ and remaining pair of electrode perpendicular to the initial pair was connected to the resistance port of the Dr. DAQ. Dr. DAQ was in turn connected to the computer. The source of electric current was from ADC card (O-OSCILLOSCOPE) and Dr. DAQ. By using associated Pico scope software, different frequencies of current (maximum $20 \mathrm{kHz}$ ) were passed. Output (R) was monitored by Pico-scope. We measured impedance as ECIT- Electrical Imp Tomography or spectroscopy to provide time dependent information.

\section{Experimental design to find the safe current limit (optimization experiments)}

We conducted a series of experiments at the frequency of $100-500 \mathrm{~Hz}, 1 \mathrm{kHz}, 2-10,10-20 \mathrm{mHz}$ (1-6 mV; 0.5-3 mA) for microsphere (for 1-15 min duration). At higher frequency (10-20 mHz) and duration (10-15 min), microsphere ruptured within $15 \mathrm{~min}$ and huge entropy and heat energy generated/produced.

\section{Optimal limit}

We calculated optimal frequency and time for creating onco-thermia and cell lysis in cell suspension and tissues and found the frequency of $100 \mathrm{~Hz}(1-6 \mathrm{mV} ; 500$ $\mu \mathrm{A}$ or $0.5 \mathrm{~mA}$ ) to a maximum frequency of $1 \mathrm{kHz}$ (at 500 $\mu A$ ) given for 1 minute to be optimal where cell mem- brane remained intact without disruption and lysis and beyond 3 minutes, we observed fall in the impedance, cell disruption and death.

\section{Methodology of protein extraction and assay}

Levels of HSP90, beta-actin and histone proteins $\mathrm{\gamma H} 2 \mathrm{AX}, \mathrm{H} 3, \mathrm{H} 3 \mathrm{~K} 9 \mathrm{ac}$ was evaluated by western blotting. HSP90 stands for Heat Shock Protein 90 that is a cellular protein involved in stabilizing other proteins during heat stress. This particular protein was chosen for analysis considering the heat generated during the experimental setup. Gamma H2AX defines a post-translationally modified form of histone variant H2AX that depicts genomic instability. $\mathrm{H} 3 \mathrm{~K} 9 \mathrm{ac}$ is a post-translationally modified form of histone H3, acetylated at Lysine 9 position. This particular PTM was chosen to analyse any global changes in the chromatin architecture since acetylated $\mathrm{H} 3 \mathrm{~K} 9$ is associated with active transcription and de-condensed form of chromatin. Briefly, the tumor tissues subjected to biophysical experiments $(100 \mathrm{~Hz}$ and $1 \mathrm{kHz}$ ) and the tissues not subjected to low frequency current (Control) were stored at $-80^{\circ} \mathrm{C}$. Tissues $(100 \mathrm{mg}$ ) and controls were also taken from the tissues at room temperature (recently sacrificed within an hour). The tissue samples were lysed by crushing in liquid nitrogen (fine powdered) followed by homogenization at $4{ }^{\circ} \mathrm{C}$ in 2 $\mathrm{ml}$ lysis buffer (20 mM Tris-HCl pH8, 2 mM EDTA pH 8, 1 $\mathrm{mM}$ Sodium Orthovanadate, $20 \mathrm{mM} \beta$-glycophosphate, $1 \mathrm{mM} \mathrm{NaF}, 1 \mathrm{mM}$ PMSF, $1 \mathrm{mM}$ DTT $10 \mathrm{mM}$ EGTA, 0.1\% Triton-X100, $5 \mathrm{mM} \mathrm{MgCl}$ and a protease inhibitor 10 $\mu \mathrm{g} / \mathrm{ml}$ Leupeptin) and incubated for 30 minutes on ice. The resultant cell extract was centrifuged for $20 \mathrm{~min}$ at $15,000 \mathrm{~g}$, after which the supernatant (total cell lysate) was collected. From pellet, histone proteins were extracted with $0.2 \mathrm{M} \mathrm{H}_{2} \mathrm{SO}_{4}$. The Total Cell Lysate (TCL) and histone proteins were boiled in Laemmli sample buffer and subjected to $10 \%$ and $18 \%$ polyacrylamide gel electrophoresis respectively. The separated proteins were transferred to a Poly Vinylidene Di-Fluoride (PVDF) membrane (Millipore, Bedford, MA, USA) and blocked for 1 hour with 5\% Bovine Serum Albumin (BSA) in TrisBuffered Saline (TBS) containing 0.1\% Tween 20 for H3, $1 \%$ BSA in $0.1 \%$ TBST for $\mathrm{YH} 2 \mathrm{AX}$ and H3K9Ac. $5 \%$ milk in $0.1 \%$ TBST was used for HSP90 and beta-actin. After blocking membranes were probed using mouse anti-H3 antibody (1:1000 dilution in 1\% BSA-TBST), anti-H2AX antibody (1:4000 dilution in 1\% BSA-TBST), anti-beta actin antibody (1:10000 dilution in 1\% BSA-TBST), Anti HSP90 antibody (1:1000 dilution in 1\% BSA TBST) and anti H3K9Ac antibody (1:1000 dilution in 1\% BSA TBST) overnight at $4{ }^{\circ} \mathrm{C}$. After three washes with $0.1 \%$ Tween 20-TBS, the blots were incubated for 1 hour with HorseRadish Peroxidase (HRP) conjugated anti-rabbit IgG (1:8000 dilution in 5\% BSA TBST) or anti-mouse IgG (1:5000 dilution in 5\% BSA TBST), again washed three times with $0.1 \%$ Tween 20 -TBS, visualized with Femto Enhanced Chemi-Luminescence (ECL) (ThermoScientific) and exposed to X-ray film, which was developed using 


\section{Flow Diagram of the Entire Experiment and the Results}

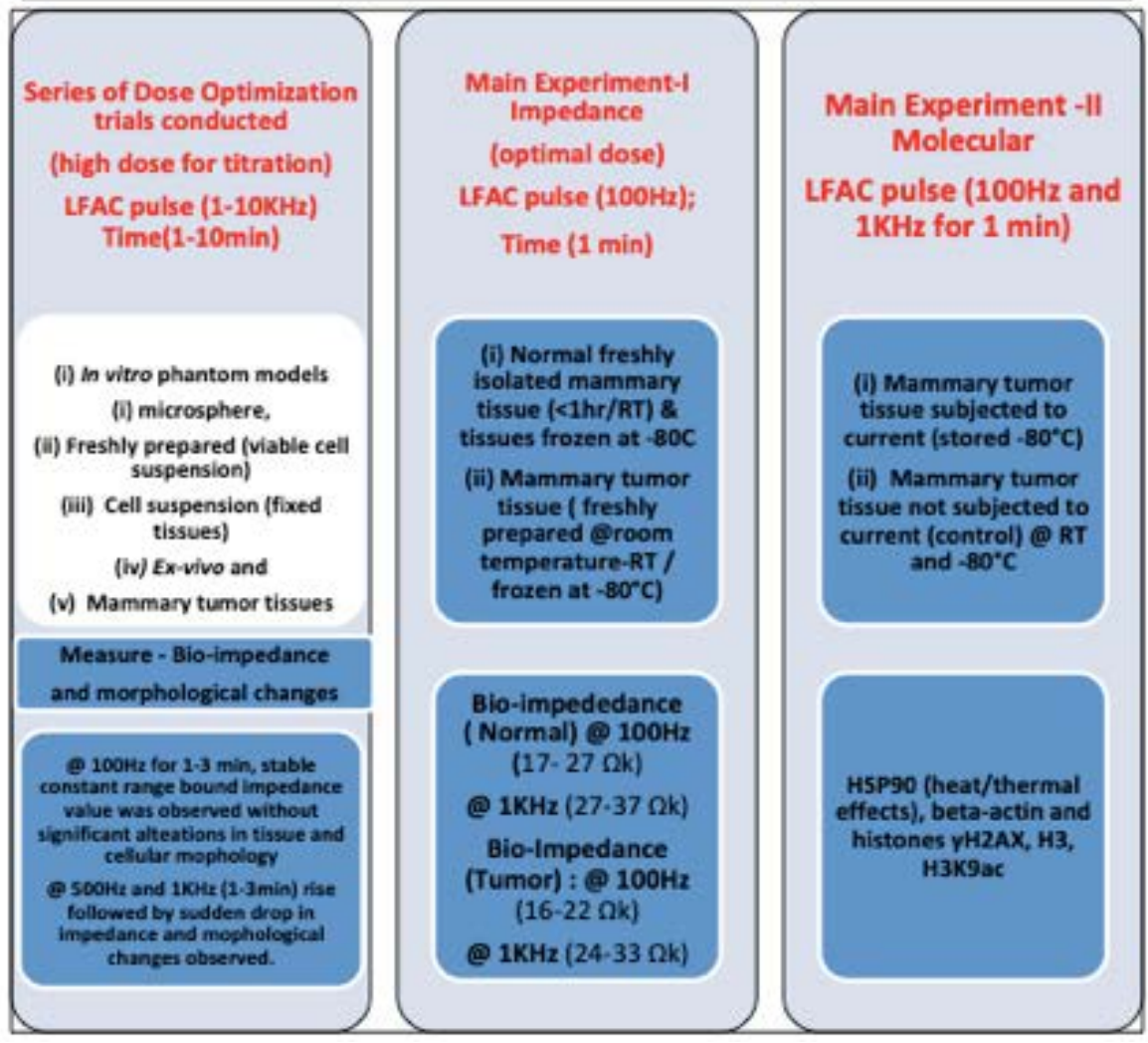

Figure 1: Flowchart diagram of experimental set up with results.
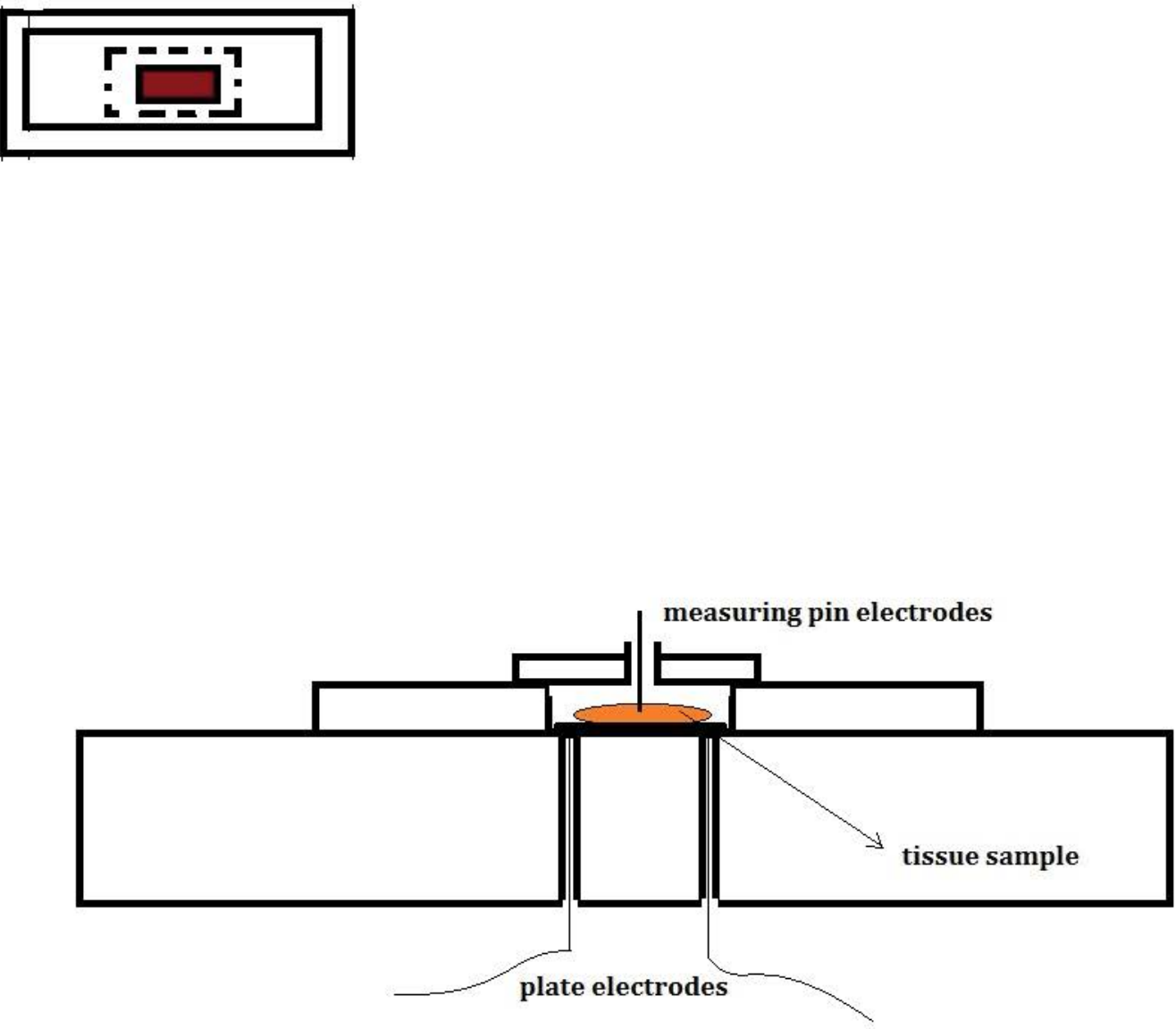

Figure 2: Diagrammatic set up for impedance measurement of cell suspensions and tissue samples. 
an automatic processor. The results were normalized to the baseline optical densities obtained with untreated cells.

\section{Results}

\section{Bio-impedance of cells and tissues}

The flowchart presented in Figure 1 depicts the experimental setup of the study and Figure 2 highlights the set up for impedance measurement of cell suspensions and tissue samples. We have highlighted the correlation between impedance and frequency in the flowchart provided as Figure 1, as well as the range determined is mentioned in the results below. The output bio-impedance ( $\mathrm{Z}$ ) was measured at an interval of 3 minutes during the passage of electric current at a frequency of $1-10 \mathrm{kHz}$ for the time period of 1 to 10 minutes. Cell disruption was observed at various time points, when $1.0 \mathrm{kHz}$ or lower frequency was passed though cell suspension beyond 3 minutes duration and at frequency of more than $10 \mathrm{kHz}$ within 1 minute. We observed increase in the tissue bio-impedance (decrease in the capacitance or conductance) value as the frequency of the applied current was gradually increased i.e., bio impedance value for normal tissue was (range 17- $27 \Omega \mathrm{k}$ ) at $100 \mathrm{~Hz}$ applied for a period of 1 minute and it increased to (range $27-37 \Omega \mathrm{k}$ ) at $1 \mathrm{kHz}$. We observed lower impedance value of (range 16-22 $\Omega \mathrm{k}$ ) at $100 \mathrm{~Hz}$ and (range 24-33 $\Omega \mathrm{k}$ ) at $1 \mathrm{kHz}$ for cancer tissue. The median value of impedance for normal tissue was $34.14 \Omega \mathrm{k}$ and for cancer tissue, it was $27.33 \Omega \mathrm{k}$ recorded at $1 \mathrm{kHz}$. Electrical conductivity (falling Z) increased initially when tissue was subjected to the passage of low frequency current compared to the impedance value (resting or recover state) when the passage of the current was stopped. We also observed a sudden fall in impedance value from $40.44 \mathrm{~K} \Omega$ (Max) to $16.1 \mathrm{~K} \Omega$ (Min) when the same low frequency $(1 \mathrm{kHz})$ was applied beyond certain duration i.e., 3 minutes or frequency was increased beyond $10 \mathrm{kHz}$. This is because the cells were not able to withstand such high frequency. The cells were able to withstand $500 \mathrm{~Hz}$ frequency with 6 $\mathrm{mA}$ of current for 1 minute very well. They remained intact without any morphological changes. The electrical conductivity also did not alter much and the impedance remained high. So, based upon our observation, we considered the safe range between $100 \mathrm{~Hz}$ (minimum) to $1 \mathrm{kHz}$ (maximum) of 1 minute duration as the baseline standard for our study. Thus, in our actual experiments, we recorded impedance value for different cell milieu at 1 min of current flow (range bound, consistent, lowest for fresh cell, highest for microsphere).

\section{Effect of low frequency pulsed electric current on unstained microsphere, stained microsphere and cell suspension}

Normal microspheres simulating biological cells are shown in Figure 3. No abnormal morphological change and lysis was observed at low frequency $(100 \mathrm{~Hz})$ except some increase in size or swelling possibly due to heating effect (Figure 4). While at higher frequency $(500 \mathrm{~Hz}-1$

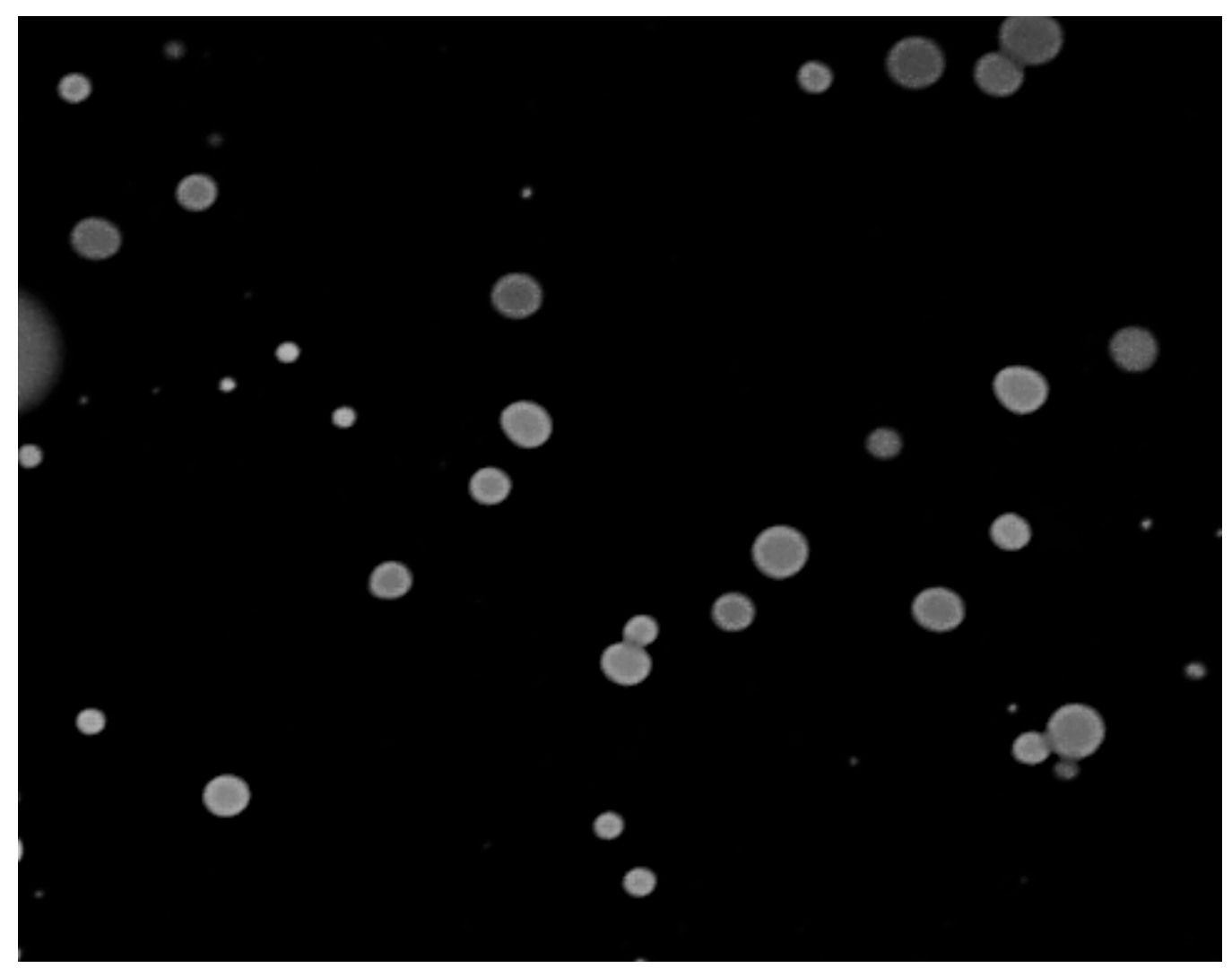

Figure 3: Stained Microsphere of different size prepared for phantom cell studies. 


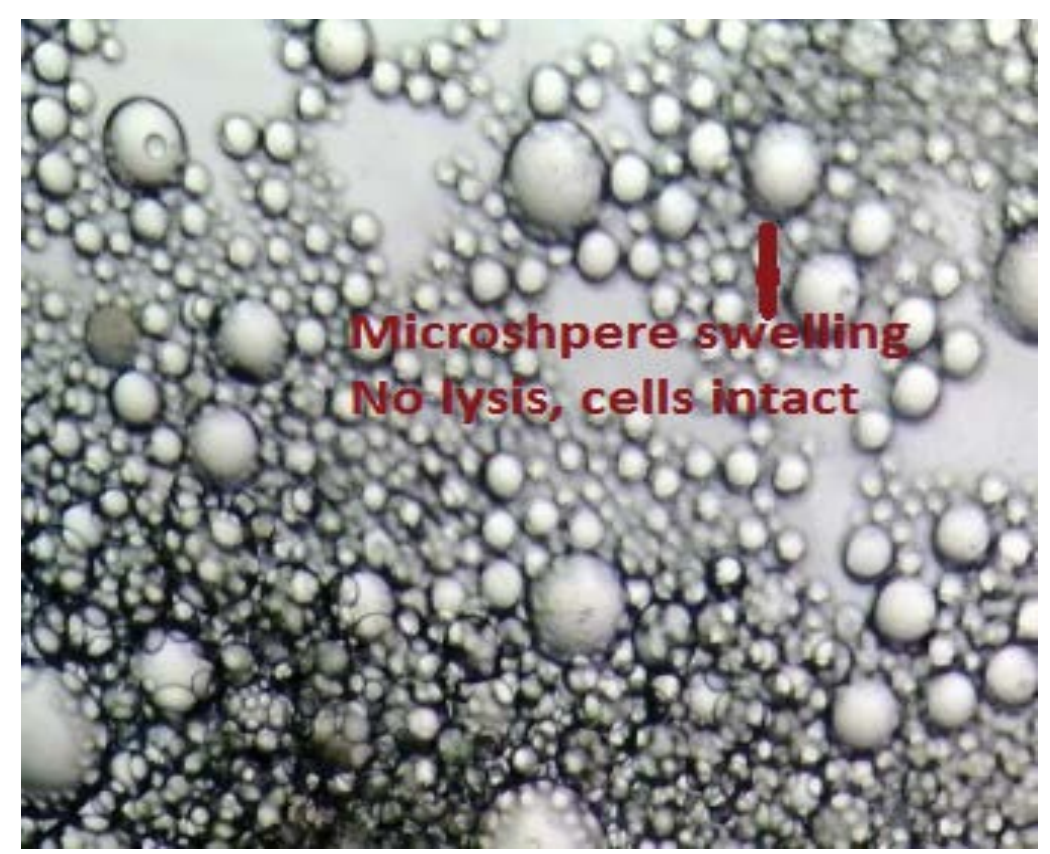

Figure 4: Microsphere (phantom cell suspension model) after 30 seconds of passing low frequency current $(500 \mathrm{~Hz})$. Representative image showing the impact of low frequency current through microsphere.

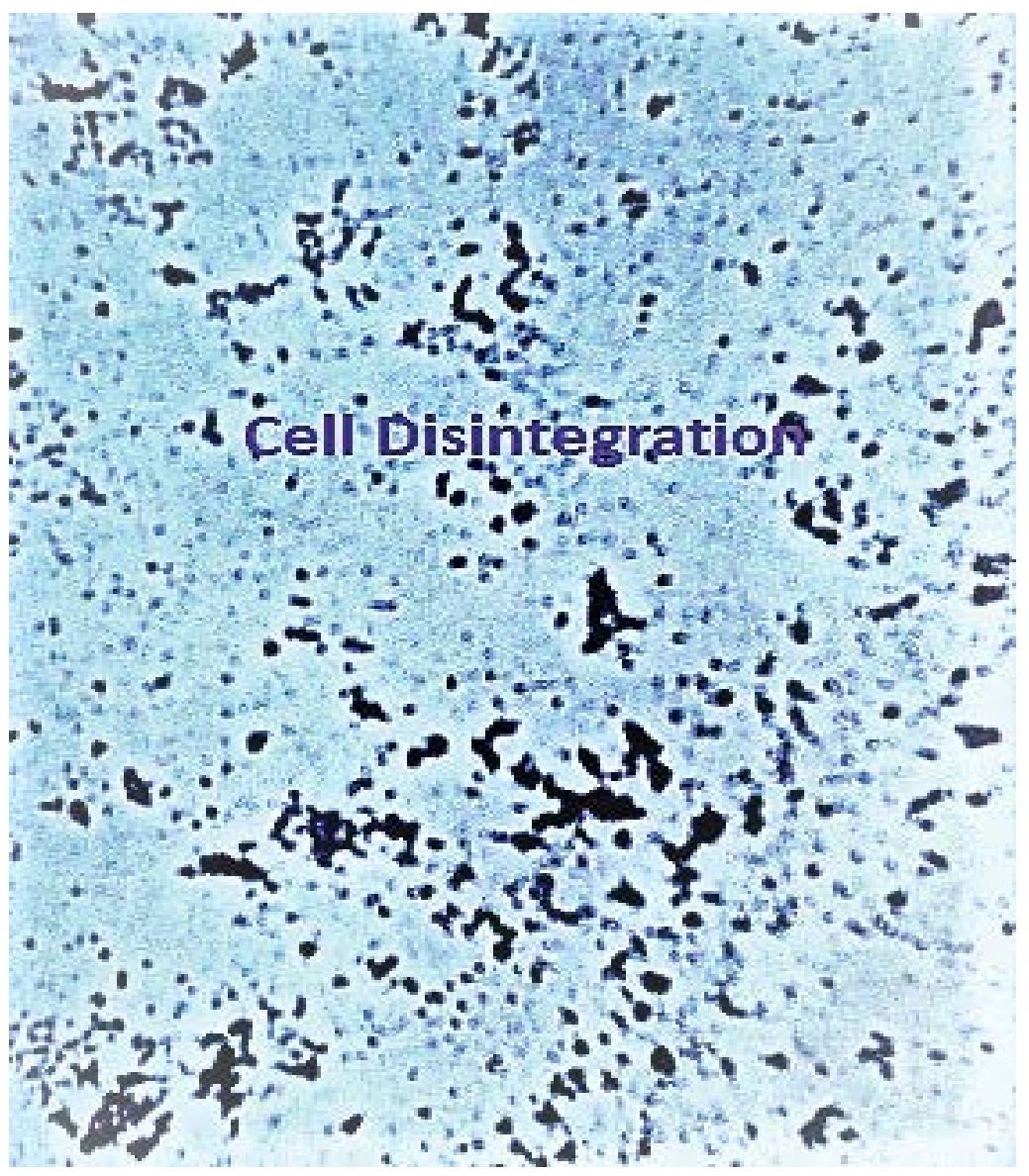

Figure 5: Biological cell suspension (cancer cell) after 60 seconds of passing low frequency current Representative image showing showing cell rupture and disruption, where impedance value also correspondingly decreased.

$\mathrm{kHz}$ ), applied for more than 1 minute, increased cell swelling, shrinkage or rupture and disintegration was noted (Figure 4, Figure 5 and Figure 6). However, high degree of stability at relatively higher frequency (when microspheres were stained) was observed signifying high entropy. The stability of microspheres increased when stained with acridine orange. Blood samples (RBC's) as well as cell suspension and microspheres underwent similar morphological changes when subjected to exogenous stress such as low frequency electric current. 


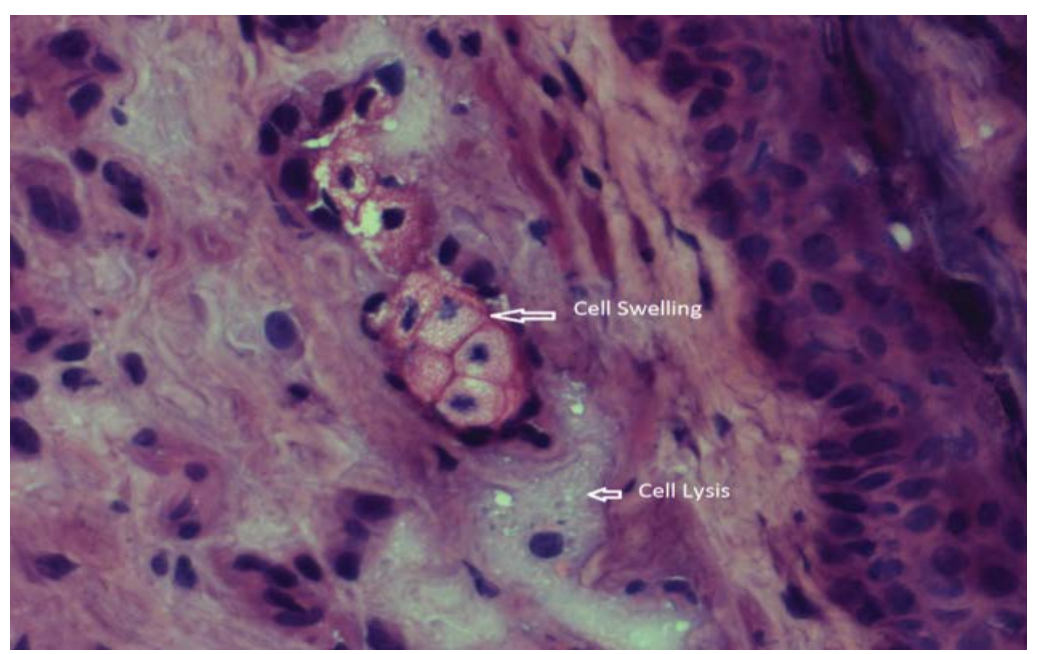

Figure 6: Biological cell (cancer cells) showed cell injury due to the passage of low frequency current for more than 60 seconds duration. Representative image showing a microscopic picture depicting cytoplasmic swelling, membrane rupture and nuclear fragmentation.

Enormous amount of heat energy or entropy was created when the microspheres were subjected to low frequency electrical stress for longer duration, which melted the plastic container in which it was contained. Therefore, microsphere model not only defined the safe and effective dose of low frequency electrical current to be applied during in vivo experimentation involving the cancer cells and tissue but also proved to be a robust model to observe the biological and physical response.

\section{Molecular study results}

We standardized the western blotting technique for histones, beta-actin, HSP9O to see the effect of electrical energy on the cancer tissues. We did not observe any alterations in the level of unmodified core histone $\mathrm{H} 3$ histone, genome instability marker $\mathrm{\gamma H} 2 \mathrm{AX}$ and global alterations of histone mark H3K9ac. This showed the effect of low frequency electrical current on the cancer tissue resulting in cell death/rupturing by inducing energy instability within without genotoxicity and epigenetic modifications (Figure 7a and Figure 7b). We have included the densitometry analysis of the western blots as Figure 7c.

\section{Discussion}

The role of exogenous or endogenous stress in modulating cancer microenvironment is being increasingly appreciated. The proliferating cancer cells interact constantly with their surrounding stroma and the microenvironment. In addition to various exogenous modalities that alters bioelectric properties of the cells, the cell membrane and the tissues, irreversible electroporation that is application of short pulses of high voltage direct current has also been utilized as a therapeutic technique with some survival benefits but not as a diagnostic tool. However, tissue heterogeneity, persistence of viable malignancy, modulation of immune system, inflammatory response, and epigenetic modifications/genotoxicity remains areas of concerns, and that need further investigation. We believe the recurrence rate will remain high unless cell-specific safe and optimal dose of current specific for specific cells and tissue types, tumor size and grade is titrated and applied under electroporation. Concurrently, one also would require examining its thermal effects on the tissue, which is a subject of controversy as of now. Work is in progress across the world to devise alternate approaches in cancer theranostics using various safer energy sources (other than radiotherapy) such as nanobased dye or agents, electro-hyperthermia, irreversible electroporation using short high voltage direct current pulse and high frequency current to create onco-thermia to kill (apoptosis, death, necrosis) the proliferating cancer cells or disrupt their aggregation as well as image the cells concurrently [25-27,40].

In this study, we manipulated the transmembrane potential, dielectric properties and entropy of both the dead and the viable cells and cancer tissues by applying a low frequency $\mathrm{AC}$ current upto $1 \mathrm{mHz}$, it produced heat as per the laws of thermodynamic as well as induced ionic flux changes. We measured changes in the bioimpedance of tissue or cell suspensions as a function of cells electrical property at a certain calculated optimal low frequency current and at the same time disrupted tumor microenvironment by applying higher stresses. Different responses were measured in normal and cancer cells and tissues i.e., in vitro and freshly prepared viable cell suspensions (freshly prepared samples exvivo) and tissues samples. Tumor cells or the excised tissue was subjected to low frequency electric current, an external stimulus, in order to modulate tumor microenvironment so that the underlying pathological as well as physiological phenomena could be studied and understood. We intended to characterize the electrical response of a cell cluster, tissues and also observe the effect of electric stress, heat (joule effect) on normal and cancer cells and tissues. The DNA damage, cell lysis 
(A)

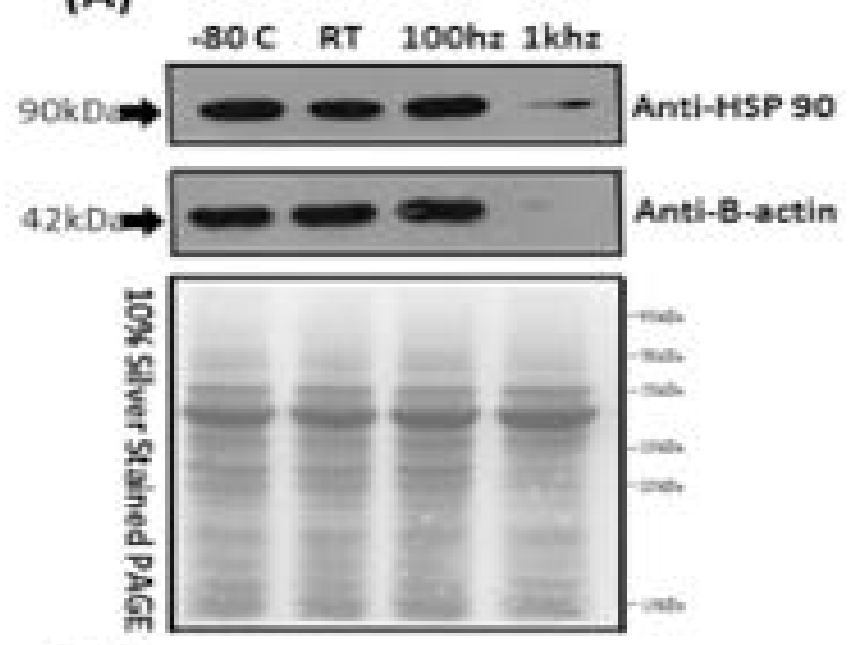

(B)

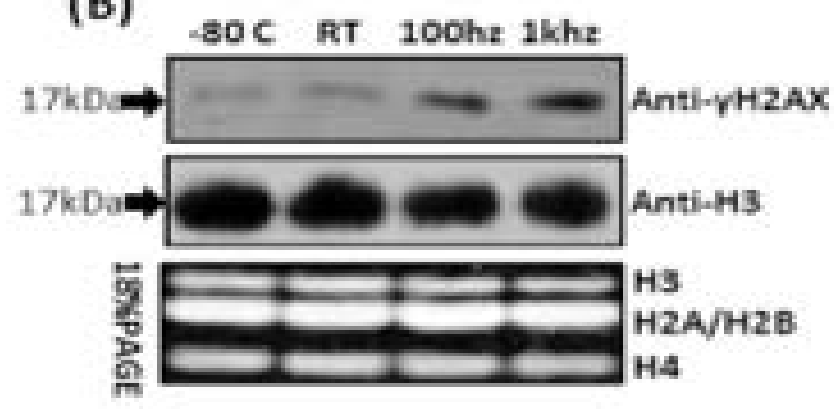

(C)

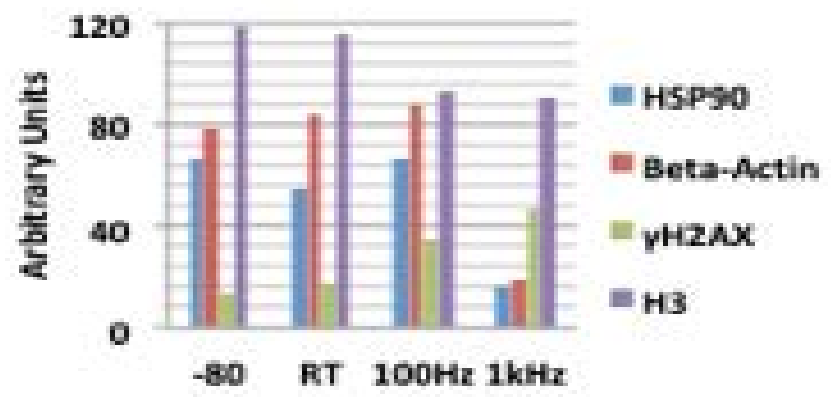

Figure 7: Levels of cellular and nuclear proteins in response to low frequency electrical current. Levels of (a) Beta-actin and HSP9O (heat shock proteins); (b) Histone modifications H3K9ac and $\mathrm{yH} 2 \mathrm{AX}$ and total histone $\mathrm{H} 3$ in cancer tissue samples (in-vitro model) following the passage of low frequency electrical current. Total cell lysate on $10 \%$ silver stained gel as well as core histones $\mathrm{H} 2 \mathrm{~A}, \mathrm{H} 2 \mathrm{~B}, \mathrm{H} 3$ and $\mathrm{H} 4$ on PVDF membrane depict equally loaded protein levels across all lanes and; (c) Graph depicts densitometric analysis of protein levels.

or deaths and other epigenetic and surface protein changes triggered by the extrinsic biophysical electrical (heat or photonic energy) were also be studied. The physics of the system used is based upon the fact that when electric current is conducted through a conducting material, resistance results in a dissipation of energy, usually in the form of heat, thus it can have many effects, notably heating, but they also modulate cancer cells EMF and trigger change in ionic flux disturbance, cell capacitance, permittivity and conductance. We also know that the living cells are energetically active and have self-entropy which is maintain throughout, therefore, stimulation of living system with external energy like current disturbs the entropy of the system leading to thermodynamic instability. Thermodynamic instability exerts physiological and biological effects by heating, up or down regulation of metabolic and other biological pathways. Morphological response at cellular level such as cell swelling, rupture and autolysis was observed besides change in the tissue electrical impedance property. Similar changes were also confirmed in the experiments conducted on the microsphere based phantom model.

Low levels of beta-actin and HSP90 proteins suggested cell surface derangement and/or apoptosis in in-vitro model and high levels of HSP90 and normal to low levels of beta-actin was seen in ex-vivo model. We did not observe any alterations in histone modification 
that was suggestive of genotoxicity. It was observed that microspheres ruptured at low frequency current when applied for certain duration of time, due to the change in the dielectric properties of the induced cells and the heat (entropy) generated within the system. The microspheres or biological cells bulged and ruptured, when tissue/cell suspension impedance fell. The tissues frozen at $-80^{\circ} \mathrm{C}$ exerted higher impedance than the fresh prepared viable tissues (kept at room temperature) since the cellular water content of the tissues kept frozen at $-80^{\circ} \mathrm{C}$ or during fixation process (in vitro) got depleted. Moreover, the cell membrane becomes inert, which reduces the electrical conductivity or permittivity thus raising the impedance. In a nutshell, we can suggest that the methodology developed to record and analyze the bio-signals can be suitably exploited in future by conducting large cells and tissue specific in vivo studies that may help in developing label free (low frequency current) diagnostic biosensor, imaging and therapeutic modalities in cancer.

\section{Conclusion}

Conventionally, various imaging modalities, labeled bio-markers or bio-sensors coated with $A B$, dye, RA agents, growth factors, fluorophores along with gold standard i.e., histo-pathological confirmations are routinely used for cancer diagnostics. So far, not many attempts have been made to exploit and manipulate the electrical or electromagnetic energy/field generated during the process of cell division to develop label free biosensor to classify and identify normal or abnormal cells. This feasibility study generated better understanding of the interactions between tissue/cell and electrical field and responses. These responses were in the form of bio-impedance, morphological change, heat production, changes in DNA and proteins. Since cellular electromagnetic and electrical properties were quantifiable, employing such properties in a large well-designed cell and tissue specific in vitro and in vivo studies can lead to a development of a robust simple cancer screening and theranostics tool in future. The challenge before the onco-scientists is to develop a safe, sensitive and reliable label free electromagnetic device or system, which doesn't have genotoxic effect, or other local and systemic untoward effects and low recurrence rate when concurrently used as a theranostics tool.

\section{Funding Source}

The research study was carried out with the institutional departmental grant; it did not receive any external or internal extramural funding.

\section{Conflicts of Interest}

The authors declare that they have no conflicts of interest.

\section{Ethical Approval}

i. All applicable international, national, and/or institutional guidelines for the care and use of animals were followed. "And all procedures performed in studies involving animals were in accordance with the ethical standards of the institution or practice at which the studies were conducted." Experimental Study was carried out in Laboratory Animal Facility (ACTREC) in strict accordance with the recommendations of the Committee for the Purpose of Control and Supervision of Experiments on Animals (CPCSEA), Ministry of Environment and Forests, Govt. of India. The protocol was approved by the Institutional Animal Ethics Committee (IAEC) of Tata Memorial Centre-Advanced Center for Treatment Research and Education in Cancer (Proposal No. 10/2012 dated, 12-08-2012, letter signed by the secretary attached). The detailed description and protocol has been discussed in material and methods.

ii. This article does not contain any studies with human participants performed by any of the authors.

\section{Data Availability}

All data generated or analyzed during this study are included in this published article (and its supplementary information file) and also available from the corresponding author on reasonable request.

\section{Acknowledgments}

Our sincere gratitude to Mr. Ravi of oxygen supply, workshop technicians for fabricating the instruments, and all the animal house staff for the safe keeps of our experimental mice during the entire period of the study. Without Mr. Parkar, Lab Assistant (Gude lab), who was instrumental in preparing cell suspension and inoculation, this work would not have been possible, we are extremely grateful for his support. Special thanks also to the summer research intern and Research Assistants of Bio-engineering lab who not only got trained but also assisted in our experiments. We are grateful to our Directors, Dr. Rajiv Sarin, Dr. Surekha Zingde, Dr S. Chiplunkar of ACTREC-TMC and other departmental heads for supporting and sanctioning departmental resources to set up project specific experimental lab much against financial and funding constraints to carry out such fundamental and translational research. They also provided time-to-time assistance to expedite the research work, without which it would not have been possible to complete this very difficult feasibility and pilot study.

\section{References}

1. Cone CD (1975) The role of surface electrical transmembrane potential in normal and malignant mutagenesis. Ann NY Acad Sci 238: 420-435.

2. Clairambault J (2013) Deterministic mathematical modelling for cancer chronotherapeutics: Cell population dynamics and treatment optimisation. 
3. Klaus K, Gabius H (1999) The application of thermodynamic principles to histochemical and morphometric tissue research: principles and practical outline with focus on the glycosciences. Cell Tissue Res 296: 443-455.

4. Tarabichi M, Antoniou A, Saiselet M, Pita JM, Dumont JE, et al. (2013) Systems biology of cancer: entropy, disorder, and selection-driven evolution to independence, invasion and "swarm intelligence". Cancer Metastasis Rev 32: 403-421.

5. Molnár J, Thornton BS, Thornton-Benko E, Amaral L, Schelz Z, et al. (2009) Thermodynamics and Electro-Biologic Prospects for Therapies to Intervene in Cancer Progression. Current Cancer Therapy Reviews 5: 158-169.

6. Sengupta A, Guha SK (1994) Multifactorial interactions in the etiopathogenesis of EPH-Gestosis a hypothesis. Med Hypotheses 43: 322-326.

7. Bradfield JJ, Kinsella JB, Mader JT, Bridges EW (1996) Rapid progression of head and neck squamous carcinoma after hyperbaric oxygenation. Otolaryngol Head Neck Surg 114: 793-797.

8. Sengupta A, Biswas P, Jayaraman G, Guha SK (1997) Understanding utero-placental blood flow in normal and hypertensive pregnancy through a mathematical model. Med Biol Eng Comput 35: 223-230.

9. Khan VR, Brown IR (2002) The effect of hyperthermia on the induction of cell death in brain, testis, and thymus of the adult and developing rat. Cell Stress Chaperones 7: 73-90.

10. Ngo MA, Sinitsyna NN, Qin Q, Rice RH (2007) Oxygen-Dependent Differentiation of Human Keratinocytes. Journal of Investigative Dermatology 127: 354-361.

11. Vander Heiden MG, Cantley LC, Thompson CB (2009) Understanding the Warburg Effect: The Metabolic Requirements of Cell Proliferation. Science 324: 1029.

12. Joyce JA, Pollard JW (2009) Micro environmental regulation of metastasis. Nature Reviews Cancer 9: 239.

13. Wezensky SJ, Cramer RA (2011) Implications of Hypoxic microenvironments during Invasive Aspergillosis. Med Mycol 49: S120-S124.

14. Davies PC, Demetrius L, Tuszynski JA (2011) Cancer as a dynamical phase transition. Theoretical Biology and Medical Modeling 8: 30.

15. Lecarpentier Y, Claes V, Vallée A, Hébert JL (2017) Thermodynamics in cancers: opposing interactions between PPAR gamma and the canonical WNT/beta-catenin pathway. Clin Transl Med 6: 14.

16. Pande S, Sengupta A, Srivastava A, Gude RP, Ingle A (2012) Re-Evaluate the Effect of Hyperbaric Oxygen Therapy in Cancer - A Preclinical Therapeutic Small Animal Model Study. PLoS One 7: e48432.

17. Sengupta A, Gupta S, Goda J, Ingle A (2018) Hyperbaric oxygen therapy, DNA damage and tumor progression. Cancer biology.

18. Prodan E, Prodan C, Miller J (2008) The Dielectric Response of Spherical Live cells in Suspension: An Analytic Solution. Biophysical Journal 95: 4174-4182.

19. Trevors JT, Pollack GH (2005) Hypothesis: The Origin of Life in a Hydrogel Environment. Progress in Biophysics and Molecular Biology 89: 1-8.

20. Fung BM, Wassil DA, Durham DL, Chesnut RW, Durham NN et al. (1975) Water in Normal Muscle and Muscle with Tumor. Biochemicaet BiophysicaActa General Subjects 385: $180-187$

21. Heileman K, Daoud J, Tabrizian M (2013) Dielectric spectroscopy as a viable bio sensing tool for cell and tissue characterization and analysis. Biosensors Bioelectron 49: 348-359.
22. Kuang W, Nelson SO (1998) Low-frequency dielectric properties of biological tissues: A review with some new insights. Amer Soc Agricult Eng 41: 173-184.

23. Al Ahmad M, Al Natour Z, Mustafa F, Rizvi TA (2018) Electrical Characterization of Normal and Cancer Cells. IEEE Access 6: 25979-25986.

24. Taghian T, Narmoneva DA, Kogan AB (2015) Modulation of cell function by electric field: A high-resolution analysis. J R Soc Interface 12: 201501.

25. Trošić I, Pavičić I (2009) Disturbance of cell proliferation in response to mobile phone frequency radiation. ArhHig Rada Toksikol 60: 109-115.

26. Luo L, Molnar J, Ding H, Xiaogui Lv, Spengler G (2006) Physicochemical attack against solid tumors based on the reversal of direction of entropy flow: An attempt to introduce thermodynamics in anticancer therapy. BMC-Diagnostic Pathology 1: 43.

27. Davies AM, Weinberg U, Palti Y (2013) Tumor treating fields: a new frontier in cancer therapy. Annals of the New York Academy of Sciences. 1291: 86-95.

28. Koreckij TD, Hill C, Azure L, Nguyen H, Kunz LL, et al. (2010) Low dose, alternating electric current inhibits growth of prostate cancer. The Prostate 70: 529-539.

29. Kulsh J (1997) Targeting a key enzyme in cell growth: A novel therapy for cancer. Medical Hypotheses 49: 297-300.

30. Klein N, Mercadal B, Stehling M, Ivorra A (2020) "In vitro study on the mechanisms of action of electrolytic electroporation (E2)". Bioelectrochemistry 133: 107482.

31. Gemert MJ, Wagstaff PG, de Bruin DM, van Leeuwen TG, van der Wal AC, et al. (2015) "Irreversible electroporation: just another form of thermal therapy?". The Prostate 75 : 332-335.

32. Zhang Y, Lyu C, Liu Y, Lv Y, Chang TT, et al. (2018) "Molecular and histological study on the effects of non-thermal irreversible electroporation on the liver". Biochemical and Biophysical Research Communications 500: 665-670.

33. Scheffer HJ, Stam AG, Geboers B, Vroomen LG, Ruarus A, et al. (2019) "Irreversible electroporation of locally advanced pancreatic cancer transiently alleviates immune suppression and creates a window for antitumor $T$ cell activation". Oncoimmunology 8: 1652532.

34. Martin RCG (2015) Use of irreversible electroporation in unresectable pancreatic cancer. Hepatobiliary Surg Nutr 4: 211-215.

35. Qin Z, Jiang J, Long G, Lindgren B, Bischof JC (2013) "Irreversible electroporation: an in vivo study with dorsal skin fold chamber". Annals of Biomedical Engineering 41: 619-629.

36. Köbbert C, Berndt A, Bierbaum T, Sontag W, Breithardt G, et al. (2008) Low-energy electromagnetic fields promote proliferation of vascular smooth muscle cells. Electromagn. Biol Med 27: 41-53.

37. Kumar S, Anand S, Sengupta A (2012) Impedance-Based Image Reconstruction of the field distribution inside the Closed Phantom Using Finite Element Method. International Journal of Advanced Research in Computer Science 1: 1-5.

38. Bhatt AN, Mathur R, Farooque A (2010) Cancer biomarkers - Current perspectives. Indian J Med Res 132: 129-149.

39. Brunetaud JM, Mordon S, Maunoury V, Beacco C (1995) Non-PDT Uses of lasers in oncology. Lasers Medical Science. 10: 3-8.

40. Tingjun Lei, Fernandez A, Manchanda R, Huang YC, McGoron AJ (2014) Near-infrared dye loaded polymeric nanoparticles for cancer imaging and therapy and cellular response after laser-induced heating. Nanotechnology 5 : 313-322. 